\title{
Economia na produção de helicônias no Estado do Rio de Janeiro
}

\author{
Economy in helicônias production in Rio de Janeiro State
}

\section{Andrezza da Silva Machado Neto ${ }^{\mathrm{I}}$ Janie Mendes Jasmim ${ }^{\mathrm{II}}$ Niraldo José Ponciano ${ }^{\mathrm{II}}$}

\section{RESUMO}

A pesquisa teve como objetivo determinar mediante o cálculo do valor presente líquido (VPL), da taxa interna de retorno (TIR) e do Payback Econômico (PBE), a viabilidade da produção de helicônias no Estado do Rio de Janeiro, bem como identificar e verificar, por meio da análise de sensibilidade, em que medida alguns fatores do sistema produtivo exercem impacto sobre a rentabilidade das regiões produtoras. Os resultados mostraram que a produção de helicônias é uma atividade lucrativa, segundo o método da TIR e considerando taxas mínimas de atratividade (TMA) de $6 \%$ e $12 \%$ para o cálculo do VPL. Porém, ocorrem limitações no setor de escoamento da produção, retardando a consolidação desse mercado. O percentual de perdas e o preço de venda do produto apresentaram-se como fatores determinantes sobre o tempo de retorno do investimento (PBE). Além disso, o preço de venda do produto foi a variável com maior efeito sobre a rentabilidade, seguida do valor da terra e do custo das mudas.

Palavras-chave: helicônia, flor tropical, viabilidade econômica e análise de sensibilidade.

\section{ABSTRACT}

The purpose of the research was to determine, through the calculation of net present value (VPL), internal return rate (TIR) and Economic Payback (PBE), the viability of heliconias production in the Rio de Janeiro State, as well as identify and verify, by means of sensitivity analysis in which extent some factors of the production system exercise impact on the profitability of the producing regions. The results showed that heliconia production is a profitable activity, according to the method of TIR and considering minimal attractiveness rates (TMA) of $6 \%$ and $12 \%$ for calculating the net present value
(VPL). However, there are limitations in the sector of produce flow, delaying the consolidation of this market. The percentage of losses and the selling price of the product presented determinant factors on the time of return on investment (PBE). In addition, the selling price of the product was the variable with greater effect on profitability, followed by the land value and the seedlings cost.

Key words: heliconias, tropical flower, economic viability and sensitivity analysis.

\section{INTRODUÇÃO}

O mercado de flores e plantas ornamentais tem se destacado no segmento produtivo agroindustrial de forma crescente, dinâmica e promissora, tanto no mercado nacional como internacional. No Brasil, o mercado distribui-se em 50\% para flores em vasos, $40 \%$ para flores de corte e $10 \%$ para plantas ornamentais (STRINGHETA et. al., 2002).

As espécies de origem tropical são responsáveis por uma parcela desse mercado, tanto a nível nacional quanto internacional. Dentre as flores tropicais mais cultivadas no Brasil, o gênero Heliconia L., único da família Heliconiaceae, merece destaque. Segundo JUNQUEIRA \& PEETZ (2002), as helicônias ocupam a terceira colocação em área cultivada no Brasil (101,8ha), sendo superadas apenas pelas rosas (426ha) e crisântemos (234ha).

'Programa de Pós-graduação em Produção Vegetal, Universidade Estadual do Norte Fluminense Darcy Ribeiro (UENF), 28013 602, Campos dos Goytacazes, RJ, Brasil. E-mail: andrezzasmachado@yahoo.com.br. Autor para correspondência.

IDepartamento de Fitotecnia, UENF, Campos dos Goytacazes, RJ, Brasil.

II'Departamento de Engenharia e Economia na Agricultura, UENF, Campos dos Goytacazes, RJ, Brasil. 
O Estado do Rio de Janeiro apresenta o seu potencial para a produção de flores tropicais, tendo como principais elementos a diversidade de clima e solos, sendo considerado como um dos principais fornecedores dessas espécies para o Sudeste, em particular para o mercado de São Paulo (VERA, 2008). Além disso, o Rio de Janeiro conta com um programa de apoio à floricultura, denominado Florescer, por meio do qual os produtores interessados em ingressar no setor da floricultura podem contar com financiamento, assistência técnica e acesso a novas tecnologias (SECRETARIADE AGRICULTURA-RJ, 2004).

$O$ exercício da floricultura tropical tem se mostrado uma alternativa bastante atraente, pelos incentivos e pela proposta de agregar renda para o pequeno agricultor. No entanto, diante das oscilações e da volatilidade dos preços dos principais produtos agropecuários, a visão empreendedora, que busca oportunidades de negócios em setores ou culturas alternativas pode ser o diferencial no êxito de um empreendimento rural, desde que antes da implementação da lavoura seja feito um estudo de viabilidade econômica.

Nesse sentido, objetivou-se avaliar a viabilidade econômica da produção de helicônias e, por meio da análise de sensibilidade, identificar e verificar em que medida alguns fatores do sistema produtivo exercem impacto sobre a rentabilidade dessa atividade nas regiões produtoras do Estado do Rio de Janeiro.

\section{MATERIAL E MÉTODOS}

A floricultura tropical é uma atividade desenvolvida por quatorze produtores no Estado do Rio de Janeiro. Destes, dez cultivam uma ou mais espécies de Heliconia spp, sendo o principal gênero, em área cultivada, dentro da floricultura tropical no Estado do Rio de Janeiro. O cultivo é realizado em pleno sol, sob um sistema de manejo integrado (insumos químicos e orgânicos), com irrigação (MACHADO NETO, 2010). Segundo este mesmo autor, trata-se de uma atividade de caráter econômico secundário e recente para a maioria dos produtores de flores tropicais (73\%), apresentando-se como uma proposta de agregação de renda para o agricultor.

Avaliação da viabilidade econômica

A viabilidade econômica do cultivo de helicônias no Estado do Rio de Janeiro foi avaliada por regiões administrativas, conforme a divisão territorial, definida pelo Censo da Floricultura no Rio de Janeiro (EMATER-RIO, 2004), e considerando um período de investimento de cinco anos.
A avaliação da viabilidade econômica foi realizada em duas etapas. A primeira etapa consistiu na elaboração do fluxo de caixa composto por todos os valores monetários das despesas (fixas e variáveis) e receitas referentes ao cultivo de helicônia, ao longo do período do investimento de cinco anos. Ademais, a partir da conclusão dos fluxos de caixas, prosseguiuse para a realização da segunda etapa da análise econômica constituída pelo cálculo dos indicadores de rentabilidade.

Todos os valores monetários empregados na análise econômica, sejam de produtos ou de insumos, foram coletados no ano de 2009, nas próprias regiões em que se situam as unidades produtoras, para identificar o real potencial econômico das alternativas testadas.

Para avaliação da viabilidade da produção de helicônias, foram utilizados, como indicadores de resultado econômico, o Valor Presente Líquido (VPL) e a Taxa Interna de Retorno (TIR), que têm como vantagem o fato de considerar o efeito da dimensão tempo dos valores monetários e o Payback Econômico (PBE) para determinação do tempo de retorno do investimento, conforme a metodologia descrita por LAPPONI (2000).

Análise de sensibilidade

A análise de sensibilidade consiste em medir em que magnitude uma alteração prefixada em um ou mais fatores do projeto altera a rentabilidade dos resultados esperados (BUARQUE, 1991).

O procedimento para se fazer uma análise de sensibilidade consistiu em escolher o indicador a sensibilizar; determinar sua expressão em função dos parâmetros e variáveis escolhidos. Por meio de um programa de computação, obteve-se os resultados a partir da introdução dos valores dos parâmetros na expressão; fez-se simulação mediante variações de um ou mais parâmetros e verificou-se de que forma e em que proporções essas variáveis afetaram os resultados finais.

Para isso, foi adotada uma expressão numérica de variação de $10 \%$, para mais ou para menos. Assim, foram tomados valores pessimistas em relação aos custos e à rentabilidade: elevação de $10 \%$ para itens de custos e redução de $10 \%$ para os itens de receitas, resultando em uma nova Taxa Interna de Retorno (TIR) para cada cultivo. O novo resultado foi então comparado com o seu valor normal, a fim de analisar a influência de tais fatores sobre a TIR do projeto, permitindo a avaliação da viabilidade do cultivo de diferentes espécies de helicônia sob condições econômicas adversas. 
Fonte dos dados

As principais espécies cultivadas no Rio de Janeiro dentro do gênero Heliconia são: Heliconia bihai, Heliconia psittacorum, Heliconia rostrata, Heliconia latispatha, Heliconia wagneriana. Essas espécies estão distribuídas em três regiões do Estado, como segue: Heliconia bihai e Heliconia psittacorum, nas regiões Metropolitana, Médio Paraíba e Noroeste; Heliconia rostrata, nas regiões Metropolitana e Médio Paraíba; Heliconia latispatha, na região Metropolitana; e Heliconia wagneriana, na região Noroeste.

Para obtenção dos dados, foi realizada a aplicação de questionário in locu, com cada produtor de helicônia do Estado do Rio de Janeiro que se dispusera a participar da pesquisa, sendo considerados nas análises os coeficientes técnicos (insumos, equipamentos, mão-de-obra) das unidades produtoras que exercem a atividade comercialmente por um período de tempo igual ou superior ao horizonte de planejamento de cinco anos, já que é o tempo médio estabelecido para a renovação do plantio dessa cultura (LAMAS, 2002). Assim, de acordo com o número de produtores descritos na tabela 1 e a disponibilidade de dados, foi amostrado um produtor de helicônias, representativo de cada região administrativa do Estado do Rio de Janeiro, em que a cultura estava inserida.

\section{RESULTADOS E DISCUSSÃO}

Os valores obtidos para a taxa interna de retorno (TIR) e o valor presente líquido (VPL) das diversas culturas analisadas encontram-se na tabela 1 . Esses indicadores revelaram que o cultivo de helicônias, considerando todas as espécies estudadas, é viável economicamente, considerando taxas mínimas de atratividade (TMA) de $6 \%$ e $12 \%$ para o cálculo do VPL, em todo Estado do Rio de Janeiro.

Os resultados apresentados na tabela 2 mostraram a viabilidade da produção de cada espécie de helicônia no Rio de Janeiro de acordo com a realidade comercial de cada região produtora. Cada região, apresentou um percentual de perdas da produção, em virtude de limitações durante o processo de escoamento da produção.

No entanto, embora as perdas cheguem a 80\% para algumas espécies como: Heliconia bihai, Heliconia psittacorum e Heliconia rostrata, a produção de helicônias mostra-se uma atividade viável financeiramente, nas condições atuais de mercado do Estado do Rio de Janeiro, apresentando valores positivos para o VPL e uma TIR superior à maior taxa mínima de atratividade (12\%), cobrindo o custo de oportunidade do capital (COC), cujo valor pode ser considerado como equivalente à taxa de juros do mercado (BUARQUE, 1991).

Tabela 1 - Descrição dos produtores de helicônias do Estado do Rio de Janeiro conforme: a região administrativa em que estão localizados (Metropolitana (M), Médio Paraíba (MP) ou Noroeste (N); a área total da propriedade e a área com Heliconia spp., em hectares (ha); as espécies de Helicônia cultivadas em cada unidade produtiva; o tempo de atuação na atividade e o mercado de distribuição, regional (r), estadual (e) e/ou nacional (n).

\begin{tabular}{|c|c|c|c|c|c|c|}
\hline Região & Produtor & Área total (ha) & $\underset{\text { (ha) }}{\text { Área com Heliconia spp. }}$ & Descrição das Espécies & $\begin{array}{c}\text { Tempo na } \\
\text { atividade (anos) }\end{array}$ & Mercado \\
\hline \multirow[t]{2}{*}{ M } & 1 & 70 & 10 & $\begin{array}{l}H . \text { bihai, H. psittacorum, } H . \\
\text { rostrata e } H . \text { latispatha }\end{array}$ & 45 & $\mathrm{r} ; \mathrm{e} ; \mathrm{n}$ \\
\hline & 2 & - & - & - & - & - \\
\hline \multirow[t]{7}{*}{ MP } & 1 & 288 & 0,30 & H. bihai e H. rostrata & 2 & - \\
\hline & 2 & 29,5 & 0,79 & H. bihai $\mathrm{e}$ H.psittacorum & 1,5 & - \\
\hline & 3 & 81,6 & 0,14 & $\begin{array}{l}\text { H. bihai, } H . \text { psittacorum e } H . \\
\text { rostrata }\end{array}$ & 4 & $\mathrm{r} ; \mathrm{e} ; \mathrm{n}$ \\
\hline & 4 & 14,4 & 0,20 & H. bihai e H. psittacorum & 2 & - \\
\hline & 5 & 264 & 0,60 & $\begin{array}{l}\text { H. bihai, } H . \text { psittacorum e } H . \\
\text { rostrata }\end{array}$ & 5 & $\mathrm{r} ; \mathrm{e} ; \mathrm{n}$ \\
\hline & 6 & 8 & 0,34 & $\begin{array}{l}\text { H. bihai, } H . \text { psittacorum e } H . \\
\text { rostrata }\end{array}$ & 3 & $\mathrm{r} ; \mathrm{e} ; \mathrm{n}$ \\
\hline & 7 & - & - & - & - & - \\
\hline $\mathrm{N}$ & 1 & 10 & 0,04 & $\begin{array}{l}\text { H. bihai, } H . \text { psittacorum е } \boldsymbol{H} \text {. } \\
\text { wagneriana }\end{array}$ & 6 & $\mathrm{r}$ \\
\hline
\end{tabular}

Fonte: Programa Florescer (SECRETARIA DE AGRICULTURA-RJ, 2004), adaptado pelos autores.

OBS.: o segundo produtor da região Metropolitana e o sétimo produtor da região Médio Paraíba não aceitaram participar da pesquisa.

Ciência Rural, v.41, n.10, out, 2011. 
Tabela 2 - Valor Presente Líquido com horizontes de planejamentos comuns e Taxa Interna de Retorno (TIR) para o cultivo de um hectare de Heliconia spp., nas Regiões Metropolitana (1), Médio Paraíba (2) e Noroeste (3) do Rio de Janeiro.

\begin{tabular}{|c|c|c|c|c|}
\hline \multirow[b]{2}{*}{ Culturas } & \multirow[b]{2}{*}{ Região } & \multicolumn{2}{|c|}{ Valor Presente Líquido } & \multirow[t]{2}{*}{ TIR } \\
\hline & & $6,00 \%$ & $12,00 \%$ & \\
\hline \multirow[t]{3}{*}{ Heliconia bihai } & $1^{\mathrm{a}}$ & $24.056,93$ & $11.505,73$ & $20 \%$ \\
\hline & $2^{\mathbf{b}}$ & $16.671,84$ & $6.996,81$ & $18 \%$ \\
\hline & $3^{c}$ & $49.038,64$ & $31.847,86$ & $30 \%$ \\
\hline \multirow[t]{3}{*}{ Heliconia psittacorum } & $1^{\mathrm{a}}$ & $189.362,27$ & $151.372,91$ & $94 \%$ \\
\hline & $2^{b}$ & $13.118,52$ & $1.683,43$ & $13 \%$ \\
\hline & $3^{d}$ & $344.056,21$ & $278.398,90$ & $118 \%$ \\
\hline \multirow[t]{2}{*}{ Heliconia rostrata } & $1^{\mathrm{a}}$ & $25.141,04$ & $12.573,07$ & $20 \%$ \\
\hline & $2^{\mathbf{b}}$ & $17.430,17$ & $6.000,63$ & $16 \%$ \\
\hline Heliconia latispatha & $1^{\mathrm{a}}$ & $14.146,84$ & $3.033,03$ & $14 \%$ \\
\hline Heliconia wagneriana & $3^{d}$ & $114.500,29$ & $87.118,94$ & $59 \%$ \\
\hline
\end{tabular}

Obs.: ${ }^{\mathbf{a}} 40 \%$ de perdas; ${ }^{\mathbf{b}} 80 \%$ de perdas; ${ }^{\mathbf{c}} 70 \%$ de perdas; ${ }^{\mathbf{d}} 20 \%$ de perdas.

A variação nos valores dos indicadores econômicos, encontrada entre as regiões produtoras do Estado para uma mesma espécie, está relacionada com o percentual de perdas, o preço do produto, a produtividade e o número de plantas por hectare.

Em geral, plantios adensados apresentam um maior número de plantas por hectare. E quando este adensamento é dimensionado, de modo que não haja interferência negativa sobre a produtividade por planta, consequentemente, obtém-se maior volume de produção e rentabilidade com um maior número de plantas por hectare (KREUZ et. al., 2006).

Além disso, a distância da região Metropolitana, onde se concentra uma grande oferta desse produto, permite com que os preços recebidos no Noroeste sejam mais elevados, viabilizando a produção de helicônias, mesmo sob elevados percentuais de perdas para algumas espécies nessa região, como revelam os dados apresentados na tabela 2.

Assim, os resultados econômicos tornamse atraentes para os produtores, o que ocasiona a entrada de novos concorrentes no mercado. Essa situação sugere que é um mercado de lucros efêmeros, pois, com a maior oferta, provavelmente, esses resultados obtidos pelos indicadores de rentabilidade diminuirão e os produtos deverão se tornar mais acessíveis.

Considerando a experiência do mercado de flores tropicais no Estado de Pernambuco (LOGES et al., 2005), há, na elaboração de um plano estratégico de ações mercadológicas, uma alternativa para que seja alcançada a máxima eficiência financeira no Estado do
Rio de Janeiro, de modo a promover as inflorescências de helicônias no mercado potencial de flores e plantas ornamentais. Dentre as ações mercadológicas mais eminentes estão: a divulgação do produto, o investimento na qualidade floral, o uso de embalagens e a competitividade.

No que se refere ao Payback Econômico (PBE), a região Médio Paraíba apresentou um tempo superior a quatro anos para o retorno do investimento, para todas as espécies cultivadas, considerando um volume de perdas da produção de $80 \%$. Todavia, esse tempo reduziu cerca de até $80 \%$ no cultivo de Heliconia bihai, quando se estimou, sobre o volume de inflorescências produzidas, um percentual de perdas de apenas $10 \%$ nesta mesma região.

$\mathrm{Na}$ região Noroeste, obtiveram-se os menores tempos para retorno do investimento no cultivo das espécies observadas, sendo estes: 3,75 anos para Heliconia bihai; 1,26 anos para Heliconia psittacorum; e 2,09 anos para Heliconia wagneriana. Nesse sentido, essa região apresenta o seu potencial, em termos econômicos, para a produção de helicônias. Associando dois importantes fatores do processo decisório da avaliação de projetos agropecuários: resultados satisfatórios dos indicadores econômicos (VPL e TIR) e menor tempo de retorno do investimento (PBE).

O maior preço do produto e o menor volume de perdas para a maioria das espécies foram os dois fatores que justificaram a rapidez no retorno dos investimentos no cultivo de Heliconia spp., na região Noroeste, se comparado com às demais regiões do 
Estado. Ademais, observa-se que, dentre os dois fatores citados, o preço do produto mostra-se como fator preponderante sobre os demais, seja nas condições atuais, de até $80 \%$ de perdas para algumas Regiões ou sob uma condição hipotética de apenas $10 \%$ de perdas da produção.

As perdas na região Metropolitana, para Heliconia bihai, correspondem à metade do percentual de hastes florais perdidas, na região Médio Paraíba. No entanto, o PBE Metropolitano (4 anos, 6 meses e 2 semanas) é inferior ao PBE da região Médio Paraíba, em apenas um mês e sete dias, . Essa pequena diferença temporal para o retorno do investimento entre duas regiões do Estado do Rio de Janeiro, que diferem significativamente entre si quanto ao volume de perdas da produção para uma mesma espécie, é um exemplo do impacto exercido pelo preço do produto. $\mathrm{O}$ preço recebido pela unidade floral de Heliconia bihai na região Metropolitana é cerca de 70\% inferior ao da região Médio Paraíba, viabilizando e proporcionando a essa região (Médio Paraíba) um PBE, em anos, próximo do obtido na região Metropolitana.

A tabela 3 apresenta os resultados da análise de sensibilidade, mostrando o efeito sobre a taxa interna de retorno da produção de helicônia decorrente de uma variação, no sentido desfavorável, de $10 \%$ nos preços de insumos, equipamentos e produtos, nas regiões produtoras no Rio de Janeiro. Observou-se que o preço do produto foi a variável de maior impacto sobre a taxa interna de retorno dos sistemas produtivos analisados. Sendo assim, a redução de $10 \%$ no preço recebido por helicônias resultaria, por exemplo, numa queda da TIR, em pontos percentuais, de $0,59,0,36$ e 0,72 nas regiões Metropolitana (1), Médio Paraíba (2) e Noroeste (3), respectivamente. Esses resultados revelam, em termos econômicos, a influência do fator preço do produto

Tabela 3 - Redução, em pontos percentuais, na Taxa Interna de Retorno (TIR) da produção de helicônias, decorrente de uma variação desfavorável de $10 \%$ no preço dos insumos e do produto, nas Regiões Metropolitana (1), Médio Paraíba (2), Noroeste (3) do Rio de Janeiro.

\begin{tabular}{|c|c|c|c|}
\hline \multirow{3}{*}{ Item } & \multicolumn{3}{|c|}{ 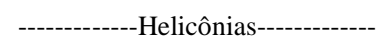 } \\
\hline & & & \\
\hline & Região 1 & Região 2 & Região 3 \\
\hline Preço do produto & $-0,59$ & $-0,36$ & $-0,72$ \\
\hline Terra & $-0,34$ & $-0,19$ & $-0,38$ \\
\hline Mudas & $-0,13$ & $-0,08$ & $-0,22$ \\
\hline Equipam. Irrigação & $-0,05$ & $-0,02$ & $-0,05$ \\
\hline Mão- de- obra & $-0,04$ & $-0,02$ & $-0,05$ \\
\hline Outros insumos e serviços & $-0,03$ & --- & --- \\
\hline Fertilizantes e corretivos & $-0,02$ & $-0,04$ & $-0,02$ \\
\hline Defensivos & --- & --- & --- \\
\hline
\end{tabular}

sobre o retorno dos investimentos na produção de helicônias no Estado do Rio de Janeiro.

A partir dos resultados dessa tabela, classificaram-se em ordem de importância os itens que mais afetaram os resultados financeiros da produção de helicônia em cada região produtora do Estado do Rio de Janeiro. Para a região Metropolitana (1), o valor da terra se destaca como o segundo item mais importante, seguido do custo das mudas. Além disso, foram importantes os custos com: equipamento de irrigação; mão-de-obra; outros insumos e serviços; fertilizantes e corretivos.

No caso da região Médio Paraíba (2), também aparece o valor da terra em segundo lugar, seguido dos custos com mudas, fertilizantes e corretivos. Empatados em quarto lugar vêm o custo do equipamento de irrigação e a mão-de-obra.

A região Noroeste, favorecida por sua posição geográfica, distante do principal centro comercial de helicônias no Estado do Rio de Janeiro, localizado na região Metropolitana, tem no preço do produto o seu principal viés. Tal item proporciona ao Noroeste rendimentos superiores às demais regiões na produção e comercialização das espécies Heliconia bihai e Heliconia psittacorum (Tabela 2). Por outro lado, o preço do produto mostra-se como item de maior impacto sobre a TIR, apresentando a maior redução em pontos percentuais em relação às demais Regiões produtoras.

Em geral, o comportamento da variável preço, num mercado de concorrência perfeita, em que há um grande número de vendedores e compradores, é determinado pelo próprio mercado, ou melhor, pela interseção da curva de demanda e oferta (CARVALHO, 2000). No caso da produção e comercialização de helicônias no Rio de Janeiro, observaram-se as características de um mercado de concorrência imperfeita, em algumas regiões do Estado, como na região Noroeste e Médio Paraíba, nas quais os produtores apresentam efeitos notáveis sobre a formação dos preços desse produto, em virtude do menor volume de produto ofertado.

Além disso, as flores podem ser classificadas como um produto cuja demanda está diretamente relacionada com o gosto do consumidor e poderão ocorrer mudanças favoráveis e desfavoráveis no consumo desse bem. Nesse sentido, as campanhas publicitárias normalmente exercem grande influência no comportamento desse tipo de consumidor.

O valor da terra e o custo das mudas ocupam, respectivamente, o segundo e terceiro lugar em importância, em relação aos impactos sobre a TIR, obtida na região Noroeste. Em seguida, vêm os custos 
com equipamento de irrigação e mão-de-obra, empatado em quarto lugar e por último os custos com fertilizantes e corretivos.

Os dados da tabela 3 demonstram que o aumento de $10 \%$ nos preços dos defensivos não exerce nenhuma influência sobre a TIR, nas regiões analisadas. Tal resultado permite uma análise discursiva sobre uma característica particular desse nicho agrícola. Segundo observações, durante o processo de coleta de dados da presente pesquisa, notou-se a preferência dos produtores em utilizar, nas áreas de cultivo, métodos alternativos de controle de pragas e doenças, baseados na agricultura orgânica, que além de apresentarem custos mais baixos e menores danos ao meio ambiente, resultam em uma baixa demanda por produtos químicos e uma parcela pequena, ou praticamente nula, dentro dos custos de produção.

Conforme os resultados apresentados, a redução na TIR da produção e comercialização de helicônia sob condições pessimistas, decorrente de uma variação desfavorável de $10 \%$ no preço dos insumos e do produto para todas as regiões consideradas, não atingiu pontos percentuais suficientes para tornar inviável a produção dessa espécie no Estado do Rio de Janeiro, segundo o método deste indicador.

\section{CONCLUSÃO}

Para as taxas de desconto consideradas (6\% e $12 \%$ ), a produção de helicônias apresentou-se economicamente viável, em todas as regiões produtoras do Estado do Rio de Janeiro. Trata-se de uma atividade lucrativa, porém dotada de algumas limitações no âmbito do processo de escoamento da produção, retardando a consolidação deste mercado. Assim, a produção de helicônias no Rio de Janeiro é um nicho de mercado em construção, sujeito a alterações nos indicadores de rentabilidade, e exigente em ações administrativas de planejamento e gerenciamento, de modo a minimizar perdas, permitindo a consolidação desse setor.

\section{REFERÊNCIAS}

BUARQUE, C. Avaliação econômica de projetos. 6.ed. Rio de Janeiro: Campus, 1991. 266p.
CARVAlHO, D.F. Padrões de concorrência e estruturas de mercado no capitalismo (Uma abordagem neoschumpeteriana). Belém: Núcleo de Altos Estudos Amazônicos/ UFPA, 2000. 142p.

EMATER - RIO, Empresa de Assistência Técnica e Extensão Rural do Estado do Rio de Janeiro. Censo da floricultura no Estado do Rio de Janeiro. Rio de Janeiro, 2004. CD-ROM.

JUNQUEIRA, A.H.; PEETZ, M.S. Os pólos de produção de flores e plantas ornamentais do Brasil: uma análise do potencial exportado. Revista Brasileira de Horticultura Ornamental, v.8, n.1/2, p. $25-47,2002$.

KREUZ, C.L. et al. Impacto da intensificação da densidade de plantio na rentabilidade em duas cultivares de macieira em Fraiburgo, SC. Revista Brasileira de Fruticultura, v.28, n.2, p.240-243, 2006. Disponível em: <http://www.scielo.br/ s c i e 1 o.ph p ? s r i p t = s c i _ a r t text \& p i d = S 0100 29452006000200019\&lng=pt\&nrm=iso >. Acesso em: 10 mar. 2010. doi:10.1590/S0100-29452006000200019.

LAMAS, A.M. Floricultura tropical: técnicas de cultivo. Recife: SEBRAE-PE, 2002. 87p. (Série Empreendedor).

LAPPONI, J.C. Projetos de investimento: construção e avaliação do fluxo de caixa, modelos em Excel. São Paulo: Lapponi Treinamento e Editora, 2000. 132p.

LOGES, V. et al. da. Colheita, pós-colheita e embalagem de flores tropicais em Pernambuco. Revista de Horticultura Brasileira, v.23, n.3, p.699-702, 2005. Disponível em: <http:/ /www.scielo.br/scielo.php?script $=$ sci_arttext\&pid $=$ S0 102 $05362005000300001 \& \operatorname{lng}=\mathrm{en} \& \mathrm{nrm}=\mathrm{iso}>$. Acesso em: 22 out. 2009. doi: 10.1590/S0102-05362005000300001.

MACHADO NETO, A. da S. Caracterização e viabilidade econômica do sistema produtivo de flores tropicais no Rio de Janeiro. 2010. 86f. Dissertação (Mestrado em Produção Vegetal) - Universidade Estadual do Norte Fluminense Darcy Ribeiro (UENF), Campos dos Goytacazes, RJ.

NORONHA, J.F. Projetos agropecuários: administração financeira, orçamento e viabilidade econômica. 2.ed. São Paulo, Atlas, 1987. 269p.

SECRETARIA DE AGRICULTURA-RJ - Estado do Rio de Janeiro. Programa de Apoio a Floricultura no Estado do Rio de Janeiro - FLORESCER, 2004. Disponível em: <http://www.agricultura.rj.gov/florescer>. Acesso em: 13 mar. 2009.

STRINGHETA, A.C.O. et al. Diagnóstico do segmento de produção da cadeia produtiva de flores e plantas ornamentais do Rio de Janeiro. Revista Brasileira de Horticultura Ornamental, v.8, n.1, p.77-90, 2002.

VERA, R.M. Diagnóstico do mercado de flores tropicais. Revista Brasileira de Horticultura Ornamental, v.14, n.1, p.35-36, 2008. 